\title{
Automatic Twitter Stance Detection on Politically Controversial Issues: A Study on Covid-19's CPI
}

\author{
Patricia D. Santos ${ }^{1}$, Denise H. Goya ${ }^{1}$ \\ ${ }^{1}$ Programa de Pós-Graduação em Ciência da Computação \\ Universidade Federal do ABC (UFABC) \\ Av. dos Estados, 5001 - Santo André - SP - Brazil \\ \{patricia.santos, denise.goya\}@ufabc.com.br
}

\begin{abstract}
Predicting the stance of social media users on a topic can be challenging, especially for unsupervised cases. In this work, retweeted posts were used as elements of user interaction, to calculate the similarities between the most active within a discussion. Stance detection for these users was performed using dimensionality reduction and clustering techniques, topic modeling using contextual embeddings, and automatic cluster labeling based on recurring terms in each group. This approach produced a small number of user clusters (between 2 and 3), with uniformity in user labeling in different bases greater than $98 \%$.
\end{abstract}

Resumo. Prever o posicionamento de usuários de mídias sociais sobre um tópico tema pode ser desafiador, especialmente para casos não supervisionados. Neste trabalho foram utilizadas postagens retuitadas como elementos de interação dos usuários, para calcular as semelhanças entre os mais ativos dentro de uma discussão. A detecção de posicionamento para esses usuários foi realizada usando técnicas de redução de dimensionalidade e clusterização, modelagem de tópicos usando embeddings contextualizados, e rotulação automática de clusters baseada em termos recorrentes em cada grupo. Esta abordagem produziu um pequeno número de clusters de usuários (entre 2 e 3), com uniformidade na rotulação dos usuários em diferentes bases superior a $98 \%$.

\section{Introdução}

As redes sociais produzem uma quantidade avassaladora de dados, representando uma valiosa fonte de informação sobre seus usuários e a sociedade em que eles estão inseridos. O Twitter é uma rede social online na qual milhões de usuários postam e interagem com mensagens, popularmente conhecidas como tweets. Um tweet pode conter até 280 caracteres e possui metadados, tais como data e horário de criação, localização em coordenadas geográficas, nome de usuário, links, hashtags, emojis e menções. Além disso, tweets são públicos, seus dados são facilmente coletáveis, eles contém poucas limitações de privacidade e seu conteúdo pode ser analisado com técnicas adequadas de mineração de texto, aprendizado de máquina e processamento de linguagem natural [D'Andrea et al. 2019].

Analisar tweets pode ser uma tarefa desafiadora por causa de seu tamanho limitado e natureza ruidosa e fragmentada, o que requer a análise de frases curtas em vez de documentos. Além disso, o conteúdo de um tweet é comumente não estruturado, podendo conter gírias, palavras de baixo calão, abreviações, hashtags, ironia, coloquialismo, e erros ortográficos ou gramaticais. Esse aspecto é ainda agravado pela esparsidade 
de dados: uma grande quantidade de termos ocorre menos de 10 vezes em um corpus [Bechini et al. 2020].

A detecção de posicionamento tem uma longa tradição no domínio dos debates políticos e ideológicos online [Mohammad et al. 2016], devido a sua aplicabilidade prática para avaliar a opinião pública, identificar grupos que se cruzam e divergem e compreender questões de interesse para diferentes comunidades de usuários [Magdy et al. 2016]. Porém, essa não é uma tarefa simples dado que conteúdos postados em mídias sociais usualmente não contêm informações contextuais em seu texto.

Trabalhos recentes sobre a detecção de posicionamento se concentram principalmente em abordagens de aprendizagem de máquina. Segundo [Darwish et al. 2020], essas abordagens têm vantagens e desvantagens: a abordagem supervisionada tem implementação simples, mas é dependente de dados de treinamento rotulados manualmente e a sua acurácia varia de acordo com a técnica de classificação escolhida e o tamanho das bases de teste e treino. Já as abordagens semi e não supervisionada, por se basearem em interações dos usuários, são mais eficazes na classificação de usuários altamente engajados (muitas vezes conseguindo obter uma classificação perfeita) e com pouca interferência humana, porém produzem resultados abaixo do ideal para usuários que raramente expressam sua opinião.

Neste trabalho apresenta-se um método de três fases para rotulação automatizada de usuários baseada em uma técnica não supervisionada de posicionamento sobre temas politicamente controversos e polarizados. No primeiro estágio ocorre a separação dos usuários com características semelhantes em clusters. No segundo estágio é feita a identificação das principais narrativas compartilhadas por eles por meio da modelagem de tópicos com base nos textos dos seus retweets, os quais são representados por meio de embeddings contextualizados, capazes de capturar os significados latentes das palavras no contexto [Samih and Darwish 2021]. O terceiro estágio consiste na rotulação automática de clusters usando uma heurística de contagem de recorrência de palavras específicas de cada cluster.

O trabalho está organizado da seguinte forma: na Seção 2 são apresentados fundamentos e trabalhos relacionados. Na seção 3, é feita a descrição da metodologia proposta. $\mathrm{Na}$ Seção 4, os resultados são discutidos. Finalmente, na Seção 5, as conclusões do trabalho e sugestões de trabalhos futuros são apresentadas.

\section{Fundamentação e Trabalhos Relacionados}

A detecção de posicionamento é um subdomínio da análise de sentimentos que visa determinar automaticamente se o autor de um texto é a favor, contrário ou neutro a uma proposição ou objeto abordados no mesmo. Ela tem aplicações generalizadas na recuperação de informações, resumo de texto e vinculação textual [Mohammad et al. 2017] e é particularmente interessante no campo das mídias sociais, pois oferece a oportunidade de identificar o posicionamento de um grande número de usuários sobre diferentes questões.

Diferentes abordagens têm sido aplicadas na tarefa de detecção de posicionamento [Küçük and Can 2020], incluindo classificadores baseados em kernel (kernelbased classifiers) [Mohammad et al. 2017], métodos de aprendizado por agrupamento 
(ensemble learners) [Zeng et al. 2016, Vychegzhanin and Kotelnikov 2019] e máquinas de vetores de suporte (Support Vector Machine-SVM) [Wojatzki and Zesch 2016]. Entretanto, abordagens de aprendizado profundo (deep learning) [Zarrella and Marsh 2016] têm ganhado espaço, sendo que trabalhos que utilizam modelos de redes neurais com representações de BERT têm apresentado resultados promissores na detecção de posição [Popat et al. 2019, Samih and Darwish 2021]. [Sirrianni et al. 2021] propõem um modelo que tenta predizer tanto a polaridade do posicionamento quanto o reconhecimento da sua intensidade. Para isso, eles utilizaram BERT e mais cinco modelos adaptados de detecção de posicionamento com alto desempenho na literatura. A aplicação de modelagem de tópicos para detecção de posicionamento também pode produzir resultados interessantes. [Lin et al. 2017] utilizaram o modelo Nonnegative Matrix Factorization (NMF) para a identificação de instâncias múltiplas de posicionamento, com o uso de rotulação automática de textos.

Existem poucos trabalhos sobre detecção de posicionamento sobre temas políticos em idiomas distintos do inglês [Vamvas and Sennrich 2020]. Como exemplo, cita-se [Rashed et al. 2021], em que os pesquisadores coletaram tweets relacionados às eleições da Turquia em 2018 e aplicaram um método não supervisionado de detecção de posicionamento. [Ebeling et al. 2020] propõem um framework para analisar e caracterizar o comportamento de grupos com posições opostas e a relação com a polarização política, usando tweets sobre a Covid-19 no Brasil. Esses autores empregaram técnicas de modelagem de tópicos, detecção de comunidade e análise de características linguísticas para determinar aspectos psicológicos nos textos.

O presente trabalho se diferencia do demais ao combinar a contagem de recorrência de palavras para rotular automaticamente o posicionamento de um usuário como contrário ou favorável em relação a um tema, com a classificação supervisionada de BERT para a modelagem de tópicos e a técnica de classificação não supervisionada proposta por [Darwish et al. 2020] para prever o posicionamento de usuários prolíficos do Twitter em relação a assuntos polêmicos em língua portuguesa.

\section{Metodologia}

A metodologia aplicada neste trabalho pode ser dividida em cinco etapas principais: coleta de dados, detecção não supervisionada de posicionamento, normalização de dados, modelagem de tópicos e rotulação automatizada. Cada uma dessas etapas é discutida nas próximas subseções.

\subsection{Coleta de Dados}

Foram coletados dados de usuários do Twitter que discutiram sobre a CPI da Covid19: uma comissão parlamentar de inquérito criada para investigar possíveis omissões do governo no combate à pandemia ${ }^{1}$. As coletas foram realizadas com a API v1.1 do Twitter $^{2}$, em tempo real, por um período de 24 horas para cada sessão de depoimentos, entre 4 de maio e 16 de julho de 2021, durante as onze primeiras semanas da CPI. Os termos e hashtags relacionados aos seus depoentes foram selecionados de acordo com os cronogramas de depoimentos divulgados na imprensa. Ao todo, foram coletados e

\footnotetext{
${ }^{1}$ http://portal.stf.jus.br/noticias/verNoticiaDetalhe.asp?idConteudo=464162\&ori=1

${ }^{2}$ https://developer.twitter.com
} 
Tabela 1. Dados Coletados para o Estudo

\begin{tabular}{|c|c|c|}
\hline \# & Termos de Busca (incluem variações sem acento) & Período \\
\hline 1 & Mandetta, Teich, depoimento, CPI, Pazuello & 04-07 Maio 2021 \\
\hline 2 & $\begin{array}{l}\text { depoimento, CPI, Anvisa, Pfizer, Antonio Barra Torres, Fabio } \\
\text { Wanjgarten, Marta Diez, Carlos Murillo }\end{array}$ & 11-14 Maio 2021 \\
\hline 3 & $\begin{array}{l}\text { CPI, CPIdaCovid, CPIdaPandemia, Pazuello, Ernesto Araújo, } \\
\text { Mayra Pinheiro, Capitã Cloroquina, CPIdoGenocidio, Kátia } \\
\text { Abreu, CPIdoCirco, DiaD }\end{array}$ & 18-21 Maio 2021 \\
\hline 4 & $\begin{array}{l}\text { CPI, CPIdaCovid, CPIdaPandemia, Mayra Pinheiro, Capitã Clo- } \\
\text { roquina, Dimas Covas, Butantan }\end{array}$ & 25-28 Maio 2021 \\
\hline 5 & $\begin{array}{l}\text { CPI, CPIdaCovid, CPIdaPandemia, Nise Yamaguchi, Clovis Arns } \\
\text { da Cunha, Zeleite Zambon, Francisco Alves, Paulo Porto Melo, } \\
\text { Luana Araújo }\end{array}$ & 01-03 Jun 2021 \\
\hline 6 & $\begin{array}{l}\text { CPI, CPIdaCovid, CPIdaPandemia, Queiroga, Elcio Franco, Wil- } \\
\text { son Lima, Claudio Maierovitch, Natalia Pasternak }\end{array}$ & $08-12 \mathrm{~J}$ \\
\hline 7 & $\begin{array}{l}\text { CPI, CPIdaCovid, CPIdaPandemia, Marcellus Campêlo, Witzel, } \\
\text { Carlos Wizard, Alexandre Marques, Ricardo Zimerman, Fran- } \\
\text { cisco Cardoso }\end{array}$ & 15-19 Jun 2021 \\
\hline 8 & $\begin{array}{l}\text { CPI, CPIdaCovid, CPIdaPandemia, Osmar Terra, Francisco } \\
\text { Emerson Maximiano, Filipe Martins, Jurema Werneck, Pedro } \\
\text { Hallal, Luis Miranda, Luis Ricardo }\end{array}$ & 22-26 Jun 2021 \\
\hline 9 & $\begin{array}{l}\text { CPI, CPIdaCovid, CPIdaPandemia, Fausto Junior, Fausto Vieira, } \\
\text { Carlos Wizard, Francisco Maximiano, Dominguetti, Davati Me- } \\
\text { dical Supply, Dominghetti }\end{array}$ & 30 Jun-02 Jul 2021 \\
\hline 10 & $\begin{array}{l}\text { CPI, CPIdaCovid, CPIdaPandemia, Regina Célia, Roberto Fer- } \\
\text { reira, Roberto Dias, Francieli Fantinato, Francieli Fontana, Wil- } \\
\text { liam Amorim, William Santana, Witzel }\end{array}$ & 06-10 Jul 2021 \\
\hline 11 & $\begin{array}{l}\text { CPI, CPIdaCovid, CPIdaPandemia, Emanuela Medrades, Amil- } \\
\text { ton Gomes, Amilton de Paula, Marcelo Blanco, Francisco Maxi- } \\
\text { miano, Cristiano Carvalho }\end{array}$ & 13-16 Jul 2021 \\
\hline
\end{tabular}

analisados onze datasets, cujo detalhamento pode ser visto na Tabela 1. O objetivo dessa coleta foi criar uma base de dados composta por conversações sobre um tema controverso, polarizado e atual. A CPI da Covid cumpre esses pré-requisitos ao retratar como a política e a ciência estão no centro da controvérsia que envolve as políticas públicas de combate à pandemia no país ${ }^{3}$.

\subsection{Detecção de Posicionamento com Base nas Interações dos Usuários}

Foi aplicado um método de detecção de posicionamento não supervisionado que busca discriminar os usuários levando em conta os usuários que eles retuítam, com base no estudo desenvolvido por [Darwish et al. 2020]. Este método foi selecionado pela sua capacidade de produzir clusters de usuários quase perfeitos e consiste nos seguintes passos:

\footnotetext{
${ }^{3}$ http://citelab.sites.uff.br/2021/05/27/analise-no-twitter-durante-primeira-semana-da-cpi-da-covidmostra-ampliacao-dos-setores-anti-bolsonaro/
} 
1. Seleção de características. A partir da aplicação de um script que selecionava todas as contas únicas que cada usuário de uma base retuitou, criou-se um filtro para localizar os usuários mais engajados na discussão. Um usuário foi considerado engajado se realizou um número mínimo de 5 retweets em cada base de dados (ou seja, ao menos 5 interações desse tipo por semana). É importante destacar que embora as bases tenham o tema CPI da Covid-19 em comum, elas foram processadas individualmente. Primeiramente, foi construído um vetor de categorias para cada usuário selecionado com base nas contas que ele retuitou. Após isso, foi calculada a similaridade do cosseno entre cada par de usuários, utilizando o vetor de categorias.

2. Redução de Dimensionalidade. Após o cálculo da similaridade, os usuários foram projetados em um espaço bidimensional utilizando o algoritmo Uniform Manifold Approximation and Projection - UMAP [McInnes et al. 2018]. A vantagem de usar o UMAP é que ele transforma dados de alta dimensão em gráficos de dispersão bidimensionais, permitindo a visualização dos dados e ao mesmo tempo preservando a sua similaridade (e possíveis clusters) [Darwish et al. 2020]. Assim, ele posiciona os usuários de forma que os semelhantes ficam mais próximos e os menos semelhantes ficam mais distantes.

3. Clusterização. Feita a projeção dos usuários em um espaço bidimensional, eles foram clusterizados utilizando o algoritmo Mean Shift. Foi utilizada a implementação da biblioteca scikit-learn ${ }^{4}$ do algoritmo.

\subsection{Normalização de Dados Textuais para Modelagem de Tópicos}

Após a detecção de posicionamento foi aplicado um script que selecionava o texto dos retweets compartilhados pelos usuários de cada cluster. Dado que, conforme discutido anteriormente, os tweets são dados textuais altamente irregulares, foi necessária a realização de uma etapa de normalização desses dados antes da modelagem de tópicos. Nesta seção são descritas as técnicas utilizadas para normalizar os textos, utilizando a plataforma NLTK $^{5}$ e expressões regulares:

1. Padronização. Detecção e remoção de links, emoticons e emojis, hashtags, pontuações, espaços e linhas em branco, 'RT' e @ (informações de usuários), números, palavras reservadas do Twitter, aspas simples e duplas e cadeias de caracteres que possuam repetições. Conversão de todas as palavras em minúsculas.

2. Tokenização. Tokenização dos textos dos tweets separados por espaços em branco, removendo também as stopwords (verbos auxiliares, preposições, artigos, pronomes e as palavras mais frequentes) e palavras com menos de três caracteres.

3. Remoção de textos duplicados.

\subsection{Modelagem de Tópicos}

Partindo da suposição de que os usuários de cada cluster teriam predominantemente a mesma posição (contrária ou a favor) em relação à CPI, foi realizada a modelagem de tópicos separadamente nos textos dos retweets compartilhadados pelos usuários de cada agrupamento majoritário e em cada base coletada.

\footnotetext{
${ }^{4}$ https://scikit-learn.org/stable/modules/clustering.html\#mean-shift

${ }^{5}$ https://www.nltk.org/
} 
Representações distribuídas de palavras e documentos como embeddings (representações aprendidas de texto em que palavras com o mesmo significado têm uma representação semelhante) ganharam popularidade devido à sua capacidade de capturar aspectos semânticos [Ebeling et al. 2021]. Para a modelagem de tópicos foi utilizada a técnica BERTopic desenvolvida por [Grootendorst 2020]. Esta técnica consiste na extração de embeddings de documentos, agrupamento para a criação de grupos de documentos semelhantes com UMAP e HDBSCAN, e na geração de tópicos através da obtenção das palavras mais importantes por meio de agrupamento com TFIDF baseado em classe (c-TF-IDF). Especificamente, para a extração dos embeddings foi utilizado o modelo BERT pré-treinado em português conhecido como BERTimbau [Souza et al. 2020]. Este modelo foi selecionado por ter sido treinado com um corpus em português brasileiro (BrWaC [Wagner Filho et al. 2018]).

\subsection{Rotulação Automática dos Clusters}

Um pequeno dicionário de termos recorrentes foi elaborado a partir da identificação de conjuntos de palavras que eram usadas predominantemente por um dos grupos: contrário ou a favor. Dentre os termos prevalentes em apenas um dos agrupamentos em cada semana, foram selecionados os mais frequentes ao longo do tempo. Foi realizada uma busca dos termos do dicionário e feita uma contagem de ocorrências de palavras nos conjuntos de tópicos gerados por cada cluster de cada base. Feito isso, foi realizada a rotulação automática de cada cluster baseada no peso relativo associado à cada palavra. Palavras como 'bolsonarismo', 'bolsonarista, 'genocídio', 'genocida', 'negacionista', 'negacionismo', 'imunidade' e 'desgoverno' foram associadas ao componente favorável à CPI. O componente contrário foi composto pelas palavras 'circo', 'palhaço', 'palhaçada', 'ladrão', 'pedofilia' e suas derivações.

\section{Resultados e Discussão}

O conjunto das 11 bases gerou um total de 99.539 usuários ativos, sendo que a detecção de posicionamento foi capaz de atribuir 87.948 usuários para dois clusters majoritários em cada base. Cerca de 11.591 usuários (aproximadamente 11,6\% da amostra) não puderam ser classificados. Houve também o agrupamento de alguns usuários em um terceiro cluster minoritário em duas das bases, o que será discutido na subseção seguinte.

A Tabela 2 mostra o quantitativo de dados analisados neste estudo. É interessante notar que apesar da percentagem de usuários clusterizados variar entre aproximadamente 13 e $28 \%$ do total da amostra, eles compuseram o que se pode chamar de uma 'minoria ruidosa', sendo responsáveis por aproximadamente 38 a $52 \%$ dos retweets de cada base.

\subsection{Clusters}

A Tabela 3 sintetiza a análise sobre a quantidade de usuários em relação aos clusters das bases nas quais eles foram rotulados. Em relação à quantidade de clusters versus a quantidade de usuários (um possível indicador de engajamento durante todo o período da CPI) e considerando que cada base equivale a uma semana de funcionamento da CPI, cerca de $33 \%$ dos usuários foi rotulado em apenas uma base enquanto 62,9\% foi rotulado em mais de uma base. A relação classificação majoritária versus classificação minoritária indica a percentagem de usuários que não foi rotulada uniformemente ao longo das bases 
Tabela 2. Quantitativo dos dados analisados em cada base semanal

\begin{tabular}{ccccccc}
\hline$\#$ & $\begin{array}{c}\text { Total } \\
\text { Usuários }\end{array}$ & $\begin{array}{c}\text { Usuários } \\
\text { Rotulados }\end{array}$ & $\begin{array}{c}\text { \% Usuários } \\
\text { Rotulados }\end{array}$ & $\begin{array}{c}\text { Total de } \\
\text { Retweets }\end{array}$ & $\begin{array}{c}\text { Retweets } \\
\text { Processados }\end{array}$ & $\begin{array}{c}\text { \% Retweets } \\
\text { Processados }\end{array}$ \\
\hline 1 & 177.895 & 23.698 & 13,3 & 900.172 & 346.420 & 38,5 \\
\hline 2 & 285.114 & 37.290 & 13,1 & 1.337 .120 & 522.996 & 39,1 \\
\hline 3 & 284.305 & 41.553 & 14,6 & 1.859 .796 & 907.549 & 48,8 \\
\hline 4 & 173.293 & 30.952 & 17,9 & 1.152 .504 & 499.181 & 43,3 \\
\hline 5 & 152.620 & 27.321 & 17,9 & 942.754 & 388.648 & 41,2 \\
\hline 6 & 135.704 & 18.953 & 14,0 & 723.654 & 289.024 & 39,9 \\
\hline 7 & 101.600 & 19.914 & 19,6 & 731.591 & 283.987 & 38,8 \\
\hline 8 & 201.123 & 33.252 & 16,5 & 1.334 .689 & 579.259 & 43,4 \\
\hline 9 & 133.982 & 37.290 & 27,8 & 1.006 .766 & 522.996 & 52,0 \\
\hline 10 & 129.911 & 24.686 & 19,0 & 947.183 & 375.465 & 39,6 \\
\hline 11 & 87.060 & 15.252 & 17,5 & 536.317 & 236.925 & 44,2 \\
\hline
\end{tabular}

em que eles estiveram presentes. Esse indicativo aponta a robustez da rotulação, uma vez que a percentagem de classificações divergentes variou entre 0,44 e $1,23 \%$ da amostra.

Após pesquisar alguns perfis com classificação divergente, verificou-se a existência de possíveis bots, contas automatizadas cujo um dos possíveis propósitos é retuitar conteúdos específicos em larga escala. A fim de investigar a incidência de bots na amostra, foi utilizada a ferramenta PEGABOT ${ }^{6}$ em 182 usuários que tiveram classificação divergente. Nessa análise foram considerados apenas os usuários rotulados ao mesmo tempo como contrário ou favorável ao longo das bases estudadas. Ao final, a ferramenta retornou 45 usuários com probabilidade superior a $70 \%$ do perfil ser um bot. Esse número equivale a $25 \%$ desses usuários, ou seja, um em cada quatro usuários com classificação divergente possui mais de $70 \%$ de chance de ser um bot. Optou-se por não eliminar esses usuários da amostra pois entende-se que os mesmos são um indício da precisão dos resultados.

Tabela 3. Dados de rotulação dos usuários no conjunto de onze bases analisadas

\begin{tabular}{ccccc}
\hline $\begin{array}{c}\text { Quantidade } \\
\text { de Usuários }\end{array}$ & $\begin{array}{c}\text { Clusters } \\
\text { Rotulados }\end{array}$ & $\begin{array}{c}\text { Classificação } \\
\text { Majoritária }\end{array}$ & $\begin{array}{c}\text { Classificação } \\
\text { Minoritária }\end{array}$ & $\begin{array}{c}\% \\
\text { Divergência }\end{array}$ \\
\hline 1077 & 11 & 1072 & 5 & 0.47 \\
\hline 2264 & 10 & 2254 & 10 & 0,44 \\
\hline 3200 & 9 & 3171 & 29 & 0,91 \\
\hline 3640 & 8 & 3611 & 29 & 0,80 \\
\hline 3809 & 7 & 3763 & 46 & 1,22 \\
\hline 4422 & 6 & 4394 & 28 & 0,64 \\
\hline 5255 & 5 & 5201 & 54 & 1,04 \\
\hline 6929 & 4 & 6856 & 73 & 1,06 \\
\hline 9612 & 3 & 9502 & 110 & 1,16 \\
\hline 15.080 & 2 & 14.896 & 184 & 1,23 \\
\hline 32.600 & 1 & - & - & - \\
\hline
\end{tabular}

\footnotetext{
${ }^{6}$ https://pegabot.com.br/
} 


\subsection{Tópicos}

A modelagem de tópicos forneceu uma visão geral da organização temática dos tweets processados, revelando quanta informação está disponível e as palavras mais representativas por tópico. A Figura 1 apresenta uma amostra com os cinco maiores tópicos em cinco semanas selecionadas. É interessante notar que o BERTopic ajudou a identificar argumentos semelhantes frequentes em cada cluster com base na semelhança dos tweets, além de apontar alguns vieses políticos. Por exemplo, nas semanas 1,3 e 8 é possível identificar tópicos com termos contextualmente semelhantes nos clusters rotulados como contrários à CPI: 'governadores', 'prefeitos', 'desviou recurso federal', 'corrupção', 'dinheiro público', 'investigar desvios'. É possível também identificar narrativas semelhantes entre si nas semanas 1 e 3 nos clusters rotulados como favoráveis com os termos: 'genocida', 'cpi genocídio', 'tristeza', 'tragédia', 'omissão'. Esses resultados são coerentes com um estudo que acompanha as movimentações nas redes sociais sobre os debates relacionados à CPI desenvolvido por [Gonçalves et al. 2021].

\subsection{Rotulação de Posicionamento}

A Figura 2 mostra o resultado da rotulação dos clusters com o auxílio da modelagem de tópicos e da técnica de contagem de recorrência. Os tons mais escuros de cinza indicam os termos do dicionário que tiveram alta frequência nos tópicos. Os tons de verde (favorável) e vermelho (contrário) indicam a rotulação do posicionamento dos usuários do cluster.

A Figura 3 mostra o resultado da rotulação dos clusters de acordo com o posicionamento. Os clusters em azul foram classificados como indefinidos após a aplicação da modelagem de tópicos e inspeção visual do resultado mostrar que os termos não estavam relacionados diretamente com assuntos da CPI.

Após aplicar a rotulação nos usuários clusterizados, obteve-se o seguinte resultado: 40\% (35.183) dos usuários engajados foram rotulados como contrários à CPI e são responsáveis por 46,9\% (2.324.371) dos retuítes que repercutiram entre os usuários mais ativos, enquanto que 58,6\% (51.521) dos usuários foram rotulados como favoráveis à CPI, sendo responsáveis por 53,1\% (2.628.079) dos retuítes processados. Apenas 1,4\% (1.244) dos usuários foram rotulados como posicionamento indefinido. Esses resultados confirmam uma forte polarização entre os dois grupos. Embora o grupo favorável tenha mais participantes, quando se observa o número de retuítes, percebe-se que o grupo contrário fez em média 15 retuítes a mais por usuário.

\section{Conclusão e Trabalhos Futuros}

Este estudo mostrou uma aplicação robusta de técnicas de detecção não supervisionada de posicionamento, processamento de linguagem natural e modelagem de tópicos para a rotulação automática de clusters de usuários em temas altamente polarizados e politicamente controversos.

A aplicação da técnica de rotulação automática dos clusters pode diminuir a sensibilidade do modelo de tópicos em razão do viés da interpretação humana, embora seja dependente da escolha de termos adequados para o dicionário de termos recorrentes por grupo. Em comparação com os trabalhos relacionados, o método apresentado foi capaz de rotular consistentemente mais de $98 \%$ dos usuários engajados no debate, com baixa 


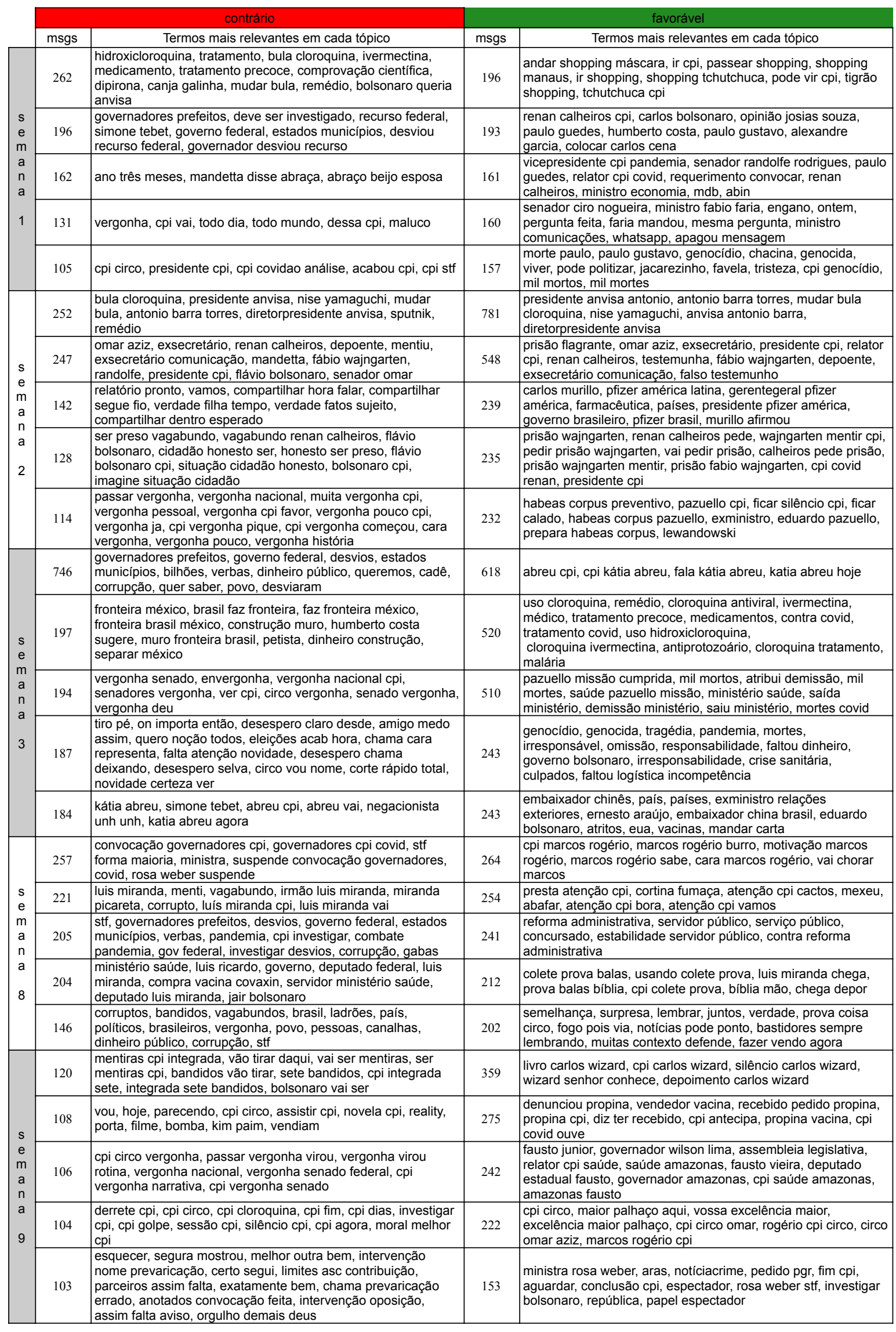

Figura 1. Exemplos de tópicos com grande número de mensagens, em cinco semanas selecionadas, com posicionamento contrário ou favorável à CPI. 


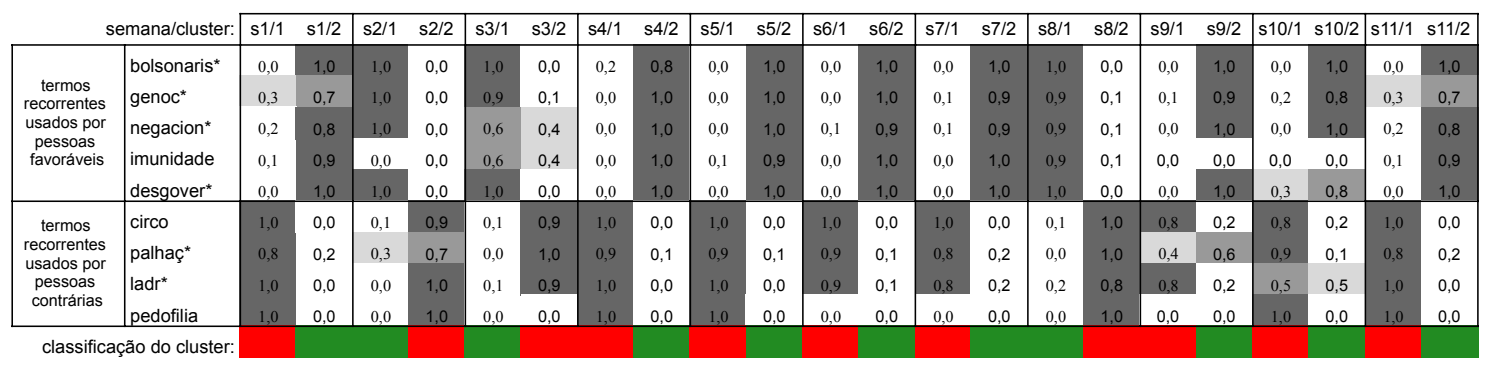

Figura 2. Prevalência de termos favoráveis e contrários em cada cluster (cinza mais escuro determina tendência para classificação).
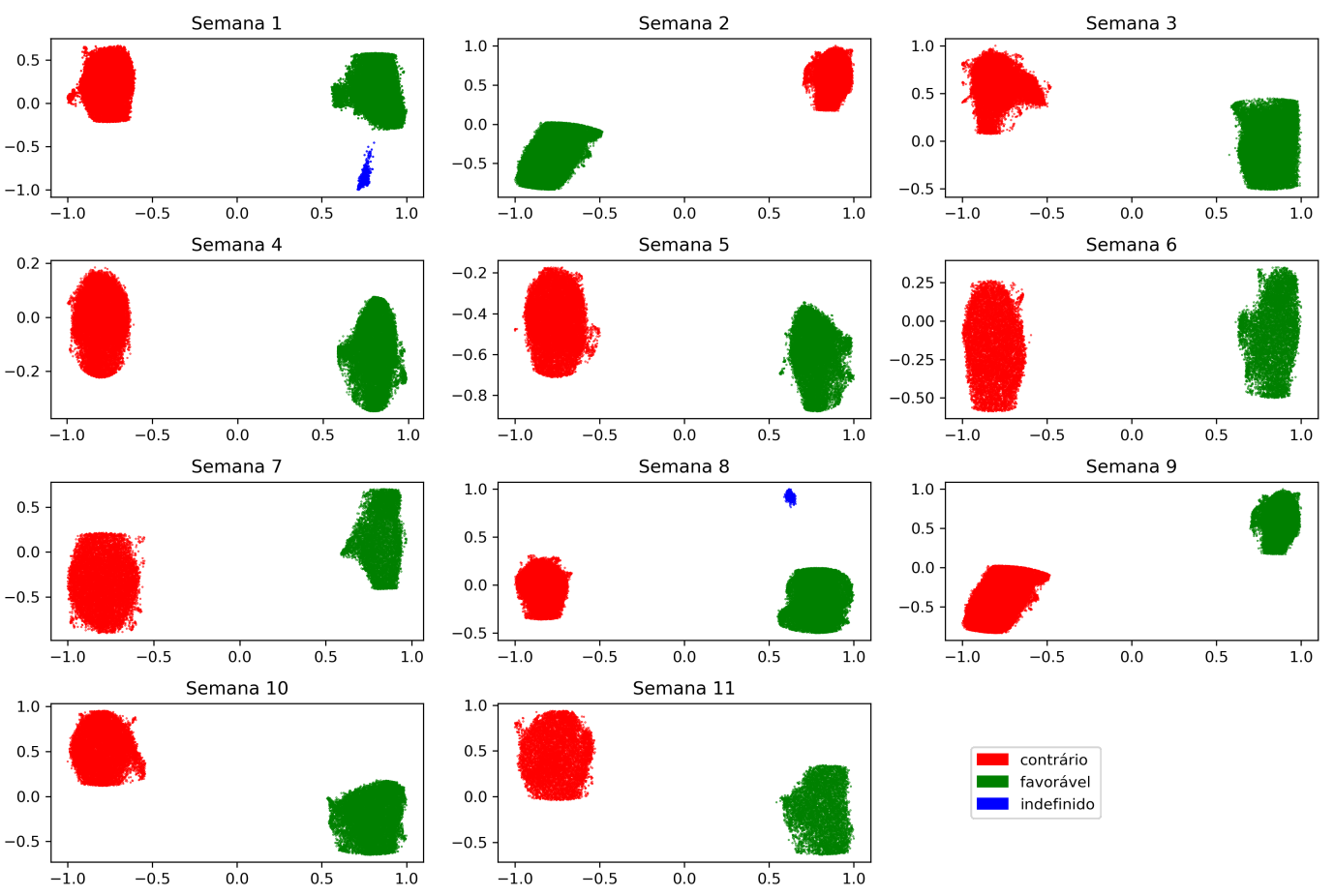

Figura 3. Rotulação automática dos clusters de usuários de acordo com o posicionamento.

intervenção humana no processo (importante para reduzir a subjetividade especialmente quando o conteúdo envolve temática polêmica).

Ao optar por coletar dados da CPI da Covid-19, obteve-se um conjunto de dados relativo a um período de discussões muito intensas sobre um tema político e altamente polarizado. A escolha de analisar apenas dados de usuários prolíficos e engajados revelou que embora eles correspondam a uma relativa minoria, ainda assim são capazes de movimentar ativamente as discussões ao serem responsáveis por cerca de $40 \%$ a $50 \%$ do volume de retweets que mais reverberaram entre os usuários processados (ou $43 \%$ da totalidade de retweets coletados). A rotulação de usuários com base em seu posicionamento frente a um assunto pode contribuir com o avanço de estudos de fenômenos em redes sociais de internet, em particular aqueles relacionados à disseminação de desinformação [Penteado et al. 2021]. 
Como trabalhos futuros, pretende-se fazer uma análise comparativa da técnica proposta neste trabalho com outras técnicas já bem estabelecidas na literatura e estender a pesquisa a fim de rotular todos os usuários das bases, utilizando técnicas de propagação de rótulos e algoritmos de classificação. Além disso, a técnica de construção do dicionário de termos recorrentes pode ser automatizada para reduzir a participação humana para apenas a conferência final dos termos. Finalmente, a técnica desenvolvida neste artigo será testada em bases que envolvam temáticas diversas, para avaliar a influência de outros fatores no processo de detecção de posicionamento não supervisionado e rotulação automatizada.

\section{Referências}

Bechini, A., Ducange, P., Marcelloni, F., and Renda, A. (2020). Stance analysis of twitter users: the case of the vaccination topic in italy. IEEE Intelligent Systems.

D’Andrea, E., Ducange, P., Bechini, A., Renda, A., and Marcelloni, F. (2019). Monitoring the public opinion about the vaccination topic from tweets analysis. Expert Systems with Applications, 116:209-226.

Darwish, K., Stefanov, P., Aupetit, M., and Nakov, P. (2020). Unsupervised user stance detection on twitter. In Proceedings of the International AAAI Conference on Web and Social Media, volume 14, pages 141-152.

Ebeling, R., Sáenz, C. A. C., Nobre, J., and Becker, K. (2020). Quarenteners vs. chloroquiners: A framework to analyze how political polarization affects the behavior of groups. In 2020 IEEE/WIC/ACM International Joint Conference on Web Intelligence and Intelligent Agent Technology (WI-IAT), pages 203-210. IEEE.

Ebeling, R., Sáenz, C. A. C., Nobre, J., and Becker, K. (2021). The effect of political polarization on social distance stances in the brazilian covid-19 scenario. Journal of Information and Data Management, 12(1).

Gonçalves, R., Oliveira, T., Toth, J., and Anchieta, W. (2021). Cpi do negacionismo: A ciência no campo das disputas político-ideológicas. http://citelab.sites.uff.br/2021/06/03/cpi-do-negacionismo-a-ciencia-no-campodas-disputas-politico-ideologicas/, Acesso em 14-08-2021.

Grootendorst, M. (2020). Bertopic: leveraging bert and c-tf-idf to create easily interpretable topics. URL https://doi. org/10.5281/zenodo, 4381785.

Küçük, D. and Can, F. (2020). Stance detection: A survey. ACM Computing Surveys (CSUR), 53(1):1-37.

Lin, J., Mao, W., and Zhang, Y. (2017). An enhanced topic modeling approach to multiple stance identification. In Proceedings of the 2017 ACM on Conference on Information and Knowledge Management, pages 2167-2170.

Magdy, W., Darwish, K., and Weber, I. (2016). \# failedrevolutions: Using twitter to study the antecedents of isis support. In 2016 AAAI Spring Symposium Series.

McInnes, L., Healy, J., Saul, N., and Großberger, L. (2018). Umap: Uniform manifold approximation and projection. Journal of Open Source Software, 3(29).

Mohammad, S., Kiritchenko, S., Sobhani, P., Zhu, X., and Cherry, C. (2016). Semeval2016 task 6: Detecting stance in tweets. In Proceedings of the 10th international workshop on semantic evaluation (SemEval-2016), pages 31-41. 
Mohammad, S. M., Sobhani, P., and Kiritchenko, S. (2017). Stance and sentiment in tweets. ACM Transactions on Internet Technology (TOIT), 17(3):1-23.

Penteado, C. L. C., Campos-Domínguez, E., Santos, P. D., Goya, D. H., Núñez, M. M., and Lázaro, M. M. (2021). Discourse and disinformation on covid-19 vaccination in spain and brazil: a case study on the twitter debate. In Palau-Sampio, editor, Contemporary Politics, Communication, and the Impact on Democracy, chapter in press. IGI Global, Hershey, PA.

Popat, K., Mukherjee, S., Yates, A., and Weikum, G. (2019). Stancy: Stance classification based on consistency cues. In Proceedings of the 2019 Conference on Empirical Methods in Natural Language Processing and the 9th International Joint Conference on Natural Language Processing (EMNLP-IJCNLP), pages 6413-6418.

Rashed, A., Kutlu, M., Darwish, K., Elsayed, T., and Bayrak, C. (2021). Embeddingsbased clustering for target specific stances: The case of a polarized turkey. In Proceedings of the International AAAI Conference on Web and Social Media, volume 15, pages 537-548.

Samih, Y. and Darwish, K. (2021). A few topical tweets are enough for effective user stance detection. In Proceedings of the 16th Conference of the European Chapter of the Association for Computational Linguistics: Main Volume, pages 2637-2646.

Sirrianni, J. W., Liu, X., and Adams, D. (2021). Predicting stance polarity and intensity in cyber argumentation with deep bidirectional transformers. IEEE Transactions on Computational Social Systems, 8(3):655-667.

Souza, F., Nogueira, R., and Lotufo, R. (2020). BERTimbau: pretrained BERT models for Brazilian Portuguese. In 9th Brazilian Conference on Intelligent Systems, BRACIS, Rio Grande do Sul, Brazil, October 20-23 (to appear).

Vamvas, J. and Sennrich, R. (2020). X-stance: A multilingual multi-target dataset for stance detection. In 5th SwissText \& 16th KONVENS Joint Conference 2020, page 9. CEUR-WS. org.

Vychegzhanin, S. V. and Kotelnikov, E. V. (2019). Stance detection based on ensembles of classifiers. Programming and Computer Software, 45(5):228-240.

Wagner Filho, J. A., Wilkens, R., Idiart, M., and Villavicencio, A. (2018). The brwac corpus: A new open resource for brazilian portuguese. In Proceedings of the eleventh international conference on language resources and evaluation (LREC 2018).

Wojatzki, M. and Zesch, T. (2016). 1tl. uni-due at semeval-2016 task 6: Stance detection in social media using stacked classifiers. In Proceedings of the 10th International Workshop on Semantic Evaluation (SemEval-2016), pages 428-433.

Zarrella, G. and Marsh, A. (2016). Mitre at semeval-2016 task 6: Transfer learning for stance detection. In Proceedings of the 10th International Workshop on Semantic Evaluation (SemEval-2016), pages 458-463.

Zeng, L., Starbird, K., and Spiro, E. S. (2016). \# unconfirmed: Classifying rumor stance in crisis-related social media messages. In Tenth International AAAI Conference on Web and Social Media. 